\title{
Gastric Cancer Screening by Combined Assay for Serum Anti-Helicobacter pylori IgG Antibody and Serum Pepsinogen Levels - The ABC Method
}

\author{
Yasuharu Yamaguchi Yasuhiko Nagata Ryuuta Hiratsuka Yoshihiko Kawase \\ Tatsurou Tominaga Shunji Takeuchi Shinya Sakagami Shusei Ishida \\ Nishitokyo Medical Association, Nishitokyo City, Tokyo, Japan
}

\section{Key Words}

Gastric cancer · Cancer screening $\cdot A B C$ method .

Helicobacter pylori $\cdot$ Pepsinogen

\begin{abstract}
Background: Helicobacter pylori (Hp) infection and gastric atrophy are both risk factors for gastric cancer. Recently it has been found that $X$-ray examination for gastric cancer screening does not have much effect on the detection rate for gastric cancer in Japan. A candidate for a new mass screening for gastric cancer, the $A B C$ method, using the combination assay of $\mathrm{Hp}$ and serum pepsinogen, was useful for identifying the development of gastric cancer in high-risk and lowrisk populations. People with higher cancer risk are recommended to receive endoscopy. The $A B C$ method was carried out as a gastric cancer mass-screening on the initiative of Nishitokyo Medical Association in Nishitokyo city from 2011. This paper reviewed the present status of gastric cancer screening using the $A B C$ method, including the latest results of our ongoing screening. Summary: We report results for 36,627 individuals from 2011 to 2013 . Among them, 16,965 received blood examination for the $A B C$ method. Of those, 8,083 planned to undergo endoscopic examination accord-
\end{abstract}

ing to stratification of the risk for the development of gastric cancer. In fact, a total of 2,911 individuals underwent endoscopic examination. Gastric cancer was detected in 65 patients, including 52 (80\%) diagnosed with early gastric cancer. The $A B C$ method was not organized screening but opportunistic screening. X-ray examination was the organized screening that was ongoing during the same period. Detection cost for 1 gastric cancer case using the $A B C$ method was cheaper than the conventional X-ray screening method $(¥ 1,267,452$ vs. $¥ 2,807,763)$. Key Messages: Although further large epidemiological studies are required, the $A B C$ method might be positioned as an effective mass screening for gastric cancer.

(c) 2016 S. Karger AG, Basel

\section{Introduction}

The discovery of Helicobacter pylori $(\mathrm{Hp})$ in 1982 has not only changed the concept of upper gastrointestinal tract diseases, but also of clinical gastroenterological practice [1]. Results of clinical and basic research accumulated over the last decade clearly demonstrate the existence of a close relationship between $H p$ infection and

\section{KARGER}

E-Mail karger@karger.com www.karger.com/dig
C 2016 S. Karger AG, Basel

0012-2823/16/0931-0013\$39.50/0
Yasuharu Yamaguchi

Yamaguchi Internal Medicine and Eye Clinic

7-16-30 Tanashicho, Nishitokyo City

Tokyo 188-0011 (Japan)

E-Mail yasuharu@ks.kyorin-u.ac.jp 
the risk of gastric cancer [2-8]. Hp infection is now recognized as the main acquired factor involved in the pathogenesis of peptic ulcer disease and chronic gastritis, and also gastric cancer [2].

Gastric cancer almost never occurs in the absence of $H p$ infection. Observation of 1,526 individuals over a period of 10 years revealed that gastric cancer was found in $5 \%$ of all individuals infected with $H p$ and in none of the uninfected individuals [5].

Serum pepsinogen (PG) was recently found to be a promising biomarker for predicting the status of the gastric mucosa [9-19]. Thus, the use of PG I concentration and PG I/II ratio for the detection of gastric atrophy was proposed. Consequently, PG may be useful in gastric cancer screening [12-19]. Recently, the combination of serum PG concentration and the presence of the $H p$ antibody has been recommended and used in some cases as a useful marker for gastric cancer screening $[1,6,20,21]$. Miki and some researchers proposed a gastric cancer screening, the ABC method, by combined assay for serum anti-Hp IgG antibody and serum PG levels $[15,21]$. The $\mathrm{ABC}$ method is classified based on serum anti-Hp IgG antibody and serum PG levels: group A (Hp (-) PG (-)), group $\mathrm{B}(H p(+) \mathrm{PG}(-))$, group $\mathrm{C}(H p(+) \mathrm{PG}(+))$, group $\mathrm{D}(H p(-) \mathrm{PG}(+))$. The risk of gastric cancer is highest in group $\mathrm{D}$, followed by that in groups $\mathrm{C}, \mathrm{B}$, and $\mathrm{A}$, in descending order $[8,15]$. If individuals are classified into a high-risk group or low-risk group through primary screening using the $\mathrm{ABC}$ method, it may be possible to recommend endoscopic examination for checking gastric cancer according to the risk level of the patients.

Gastric cancer screening using X-ray examination is widespread in Japan, a nation with high rate of stomach cancer mortality [22]. However, the present $\mathrm{X}$-ray screening system leaves much to be desired; the number of subjects screened has recently been decreasing, and the screening program itself covers less than $10 \%$ of the atrisk population. At Nishitokyo city in the northwestern Tokyo area, only $4 \%$ of residents participated in X-ray examination for gastric-cancer screenings that were organized by the local governments from 2007 to 2009 [23]. Thus, the detection rate of gastric cancer was low, from 0.14 to $0.2 \%$.

The ABC method allows stratification of the risk for the development of gastric cancer into 4 (A, B, C, and D) groups. The advantages of this examination are as follows: (1) serum PG levels do not vary greatly within 10 years or so in more than $90 \%$ of adults, (2) $H p$ infection is originally acquired in childhood in most cases, (3) the antibody titer is relatively stable in people aged 40 years or older, and (4) this examination can be performed simultaneously with a regular health checkup.

Therefore, the ABC method has been carried out as a gastric cancer mass screening on the initiative of the Nishitokyo Medical Association in Nishitokyo city since 2011.

Here, the authors review the present status of gastric cancer screening using the $\mathrm{ABC}$ method, including the latest results of our ongoing screening.

\section{Materials and Methods}

\section{Materials}

The blood examination for the ABC method is performed simultaneously with the organized regular health checkups.

Since 2011, the ABC method has been provided for Nishitokyo national health insurance system subscribers who are between the ages of 40 and 74 of even-numbered years, for example, 42, 44, and so on. Information about the ABC method was mailed to eligible individuals by the Nishitokyo city office.

$\mathrm{X}$-ray examination as the organized screening was ongoing during the same period.

\section{The ABC Method}

Serum samples collected at the time of the organized general health checkup were used to measure the serum PG I and II levels (PG I and II kit: latex agglutination method) and serum anti-Hp antibody (E-plate 'Eiken' $H p$ antibody: enzyme immunoassay method). Individuals with PG I levels of $\leq 70 \mu \mathrm{g} / \mathrm{l}$ and PG I/II ratio of $<3$ were classified as PG-positive, and those with a serum $H p$ antibody titer of $>10 \mathrm{U} / \mathrm{ml}$ were classified as $\mathrm{Hp}$-positive.

According to the ABC method [21], it is recommended that the risk for gastric cancer be stratified into 4 groups according to the anti-Hp IgG antibody titer before the eradication of $H p$ and the serum PG levels, as follows: group A $(H p(-)$ PG $(-))$, group B $(H p(+)$ PG (-)), group C $(H p(+)$ PG $(+))$, and group D $(H p(-)$ PG (+)).

To ensure correct stratification, people with a history of $H p$ eradication, treatment of proton pump inhibitors (PPIs), previous gastric resection and impairment of renal function were excluded. The subjects who had special symptoms were also excluded.

The eligible individuals in Nishitokyo city were permitted the $\mathrm{ABC}$ method only one time. Because $\mathrm{Hp}$ infection is chronic until eradication and because PG levels do not vary greatly within 10 years or so in more than $90 \%$ of adults, the ABC method generally would only be necessary once in a lifetime.

\section{Endoscopic Examination}

People in groups B, C and D are recommended to have endoscopic examination.

In Nishitokyo city, 4 hospitals and 83 clinics can perform health checkups. Of those institutions, 4 hospitals and 20 clinics can perform endoscopic examinations. Diagnosis is made solely by the performing expert endoscopist in each institution, each of whom has over 10 years of endoscopic experience. Gastric cancer is diagnosed on the basis of histological examination of forceps biopsy specimens. 
In the original $\mathrm{ABC}$ method [21], people in group A were advised to have endoscopic examinations every 5 years, those in group B every 3 years, those in group C every 2 years, and those in group D annually. However, in our screening, each endoscopist decides the time for the follow-up endoscopic examination based on the finding of the first endoscopic examination.

Endoscopic examinations following the ABC method are covered by the Japanese health insurance system.

Comparison of Detection Costs for 1 Gastric Cancer Case

We compared the cost benefit between the $\mathrm{ABC}$ method and the conventional X-ray examination for detection costs for 1 gastric cancer case.

\section{Ethics}

The ABC method was not organized screening but opportunistic screening. Therefore, the gastric cancer screening program, the $\mathrm{ABC}$ method, had to obtain approval from the ethics committee of Nishitokyo Medical Association. Participants were defined as those who provided written informed consent.

\section{Results}

Results of the ABC method in Nishitokyo city are shown in table 1. During the 3 years from 2011 to 2013, the number of individuals who received the $\mathrm{ABC}$ method was 16,965 after excluding those who met the exclusion criteria based on the information obtained from them by interview. There are no repeat individuals among the 16,965. There were 9,843 individuals in group A (58\%), 3,186 individuals in group B (19\%), 3,288 individuals in group C (19\%), and 648 individuals in group D (4\%). In group A, 961 individuals (10\%) were identified as having previous $\mathrm{Hp}$ eradication and/or taking PPI and/or having epigastric abdominal symptoms, as it turned out after the $\mathrm{ABC}$ method. All 961 in group A were advised to get the secondary endoscopic examination. Thus, 8,083 of 16,965 individuals were advised to undergo endoscopic examination, and 2,911 actually underwent endoscopy. Of the 2,911 individuals, 65 were found to have gastric cancer (detection rate of gastric cancer: $0.34 \%$ ). Of those, 13 (20\%) of them were found to have advanced cancer. The remaining 52 (80\%) had early gastric cancer. Endoscopic resection was performed in 26 of the patients (40\%), radical surgical resection was possible in 29 (45\%), and the results of remaining 10 were not received from therapeutic institutions.

Other neoplasms were detected as follows: 4 esophageal cancers, 4 MALT lymphomas, 1 duodenal cancer and 14 gastric adenomas.

Table 2 showed the comparison of detection costs for 1 gastric cancer case between $\mathrm{X}$-ray screening and the $\mathrm{ABC}$ method. Total costs of gastric X-ray screening in
Table 1. Results of the ABC method in Nishitokyo city for 3-year periods (2011-2013)

\begin{tabular}{lc}
\hline & Number of 3 years \\
\hline Screened case & $16,965(100)$ \\
Group A* & $9,843(58)$ \\
Group B* & $3,186(19)$ \\
Group C* & $3,288(19)$ \\
Group D* & $648(4)$ \\
Requiring endoscopy & $8,083(48)$ \\
Underwent endoscopy & $2,911\left(36^{* *}\right)$ \\
Detection of gastric cancer & \\
Early gastric cancer & 52 \\
Advanced gastic cancer & 13 \\
Total & 65 \\
Detection of other neoplasms & \\
Esophageal caner & 4 \\
MALT lymphoma & 4 \\
Duodenal cancer & 1 \\
Gastric adenoma & 14 \\
Total & 23
\end{tabular}

* group A $H p(-)$ PG (-), group B Hp (+) PG (-), group C Hp (+) PG (+), group D Hp (-) PG (+).

** Percentage of underwent endoscopy in requreing endoscopy.

Table 2. Comparison of detection costs for 1 gastric cancer case in Nishitokyo city

\begin{tabular}{|c|c|c|}
\hline & $\begin{array}{l}\text { X-ray from } \\
2011 \text { to } 2013\end{array}$ & $\begin{array}{l}\text { ABC method } \\
\text { from } 2011 \text { to } \\
2013\end{array}$ \\
\hline Number of subject screened, $\mathrm{n}$ & 10,259 & 16,965 \\
\hline Total cost of each screening & $¥ 46,165,500$ & $¥ 49,199,000$ \\
\hline Total cost of secondary endoscopy & $¥ 7,182,000$ & $¥ 33,185,400$ \\
\hline Number of gastric cancer, $\mathrm{n}$ & 19 & 65 \\
\hline $\begin{array}{l}\text { Cost detect one case of gastric } \\
\text { cancer }\end{array}$ & $¥ 2,807,763$ & $¥ 1,267,452$ \\
\hline
\end{tabular}

Nishitokyo city from 2011 to 2013 were $¥ 46,165,500$. In this period, 630 individuals underwent endoscopy followed by X-ray screening. Of those 630, gastric cancer was detected in 19 . Endoscopic examination costs $¥ 11,400$ per person. Detection costs for 1 gastric cancer case through X-ray screening was $¥ 2,807,763$ ( $¥ 46,165,500+¥ 11,400 \times$ $630 / 19)$. On the other hand, total costs of the ABC method from 2011 to 2013 were $¥ 49,199,000$. Endoscopy following the $\mathrm{ABC}$ method was used for 2,911 individuals. Of those 
Table 3. Comparison between X-ray screening in Japan and the ABC method

\begin{tabular}{|c|c|c|c|c|c|}
\hline & \multirow{2}{*}{$\begin{array}{l}\text { X-ray screening } \\
\text { in Japan } 2006\end{array}$} & \multicolumn{4}{|c|}{ The ABC method } \\
\hline & & $\begin{array}{l}\text { Takasaki } \\
2007\end{array}$ & $\begin{array}{l}\text { Fushimi } \\
2012\end{array}$ & $\begin{array}{l}\text { Meguro } \\
2008-2011\end{array}$ & $\begin{array}{l}\text { Nishitokyo city } \\
2011-2013\end{array}$ \\
\hline Number of subjects screened, $\mathrm{n}$ & $4,262,048$ & 16,955 & 4,981 & 23,952 & 16,965 \\
\hline Number of subjects required endoscopy, $\mathrm{n}$ & $4,27,949$ & 8,357 & 3,192 & 9,356 & 8,083 \\
\hline Rate of subjects required endoscopy, \% & 10 & 50.7 & 64.1 & 39 & 48 \\
\hline Detection rate of gastric cancer, $\%$ & 0.15 & 0.25 & 0.42 & 0.22 & 0.38 \\
\hline
\end{tabular}

2,911, gastric cancer was detected in 65 . Detection costs for 1 gastric cancer case using the $\mathrm{ABC}$ method was $¥ 1,267,452(¥ 49,199,000+¥ 11,400 \times 2,911 / 65)$. The ABC method was cheaper than X-ray screening.

No case of incidental disease or endoscopy-related infection discovered by endoscopic examination and requiring medical intervention has yet been discovered.

\section{Discussion}

Gastric cancer screening by combined assay for serum anti-Hp IgG antibody and serum PG levels, the ABC method, has been proposed by some researchers since the early 1990s [15, 21]. The ABC method has already been carried out at some work places and by some local governments [21]. Our study shows results similar to those of previous reports (table 3) [24]. From 2011 to 2013 , the number of patients who were detected as having gastric cancer through the $\mathrm{ABC}$ method was more than 3 times that detected through $\mathrm{X}$-ray screening in Nishitokyo city (table 2). Moreover, the ABC method for detection cost of 1 gastric cancer case was cheaper than $\mathrm{X}$-ray screening. Thus, our data provide supporting evidence of the efficacy of the ABC method. At the turning point of gastric cancer screening in Japan, mass screening using endoscopy following the $\mathrm{ABC}$ method for stratifying gastric cancer risk may open a new era for gastric cancer screening.

However, there are some problems with the $A B C$ method, which are shown in this report. One of the problems is that group A, the low risk group, included subjects who had received $H p$ eradication, as was also the case in previous reports [25]. Those subjects still have risk of gastric cancer. In our study, $10 \%$ in group A included subjects with the exclusion criteria such as previous $H p$ erad- ication. It is not efficient to attempt to eliminate subjects with $H p$ eradication and/or $H p$ natural disappearance and/or taking PPI only by including a careful interview before examination. Because of time constraints and because of subjects who were unclear about their own medical history, the elimination of subjects with a history of $H p$ eradication was not completely successful, and some subjects who had received $H p$ eradication were included in group A.

The low rate of secondary endoscopic examination following the $\mathrm{ABC}$ method is also a negative factor for successful gastric cancer screening. The mean rate of secondary endoscopy in this report was only $36 \%$. It is clear that an increase in the secondary endoscopic examination rate is important for determining the effectiveness of gastric cancer screening. Efforts to increase screening rates require the creation of an organized system for screening. Moreover, it is recommended that endoscopic examination be performed for subjects with cancer risk into the future. We, especially the attending doctor for each participant, have the responsibility for advising endoscopy to the subjects in groups B-D repeatedly. Therefore, partnership with local governments is crucial for development of the ABC method.

In the original practice of the $\mathrm{ABC}$ method [21], people in group A were advised to have endoscopic examinations every 5 years, those in group B every 3 years, those in group C every 2 years, and those in group $\mathrm{D}$ annually. However, there were not a few patients with advanced atrophic gastric mucosa in groups $\mathrm{B}$ and $\mathrm{C}$. Thus, it was difficult to decide the time for the follow-up endoscopic examination based on stratification according to the original $A B C$ method.

The ABC method in Nishitokyo city was performed as opportunistic screening. The ABC method for organized screening is still in question because satisfactory evidence 
showing decreased mortality rates from gastric cancer has not yet been demonstrated. Organized screening requires more responsibility for the eligibility requirement, more quality assurance, and better specificity of follow-up and evaluation. It cannot be completely denied that the participants in the ABC method received harmful effects associated with low quality. To make the $\mathrm{ABC}$ method more practical for communities, a large epidemiological study is desirable [26].

In conclusion, the $\mathrm{ABC}$ method might be premature for organized screening for gastric cancer. However, previous papers and this paper have been reporting a cancer detection rate and cost benefit better than that found using conventional X-ray mass screening. Also, subjects diagnosed as $H p$ positive may be receiving eradication through the use of the ABC method. Thus, we can expect greater ability to reduce gastric cancer incidence and mortality through the $\mathrm{ABC}$ method in future.

\section{Disclosure Statement}

The authors report no conflicts of interest.

\section{References}

1 Asaka M, Kato M, Takahashi S, Fukuda Y, Sugiyama T, Ota H, Uemura N, Murakami K, Satoh K, Sugano K: Guidelines for the management of Helicobacter pylori infection in Japan: 2009 revised edition. Helicobacter 2010;15:1-20.

2 Hatakeyama M: Oncogenic mechanisms of the Helicobacter pylori CagA protein. Nat Rev Cancer 2004;4:688-694.

3 Nomura A, Stemmermann GN, Chyou PH, Kato I, Perez-Perez GI, Blaser MJ: Helicobacter pylori infection and gastric carcinoma among Japanese Americans in Hawaii. N Engl J Med 1991;325:1132-1136.

4 International Agency for Research on Cancer: Schistosomiasis, liver flukes and Helicobacter pylori. IARC Monogr Eval Carcinog Risks Hum 1994;61:177-240.

5 Uemura N, Okamoto S, Yamamoto S, Matsumura N, Yamaguchi S, Yamakido M, Taniyama K, Sasaki N, Schlemper RJ: Helicobacter pylori infection and the development of gastric cancer. N Engl J Med 2001;345: 784-789.

6 Watabe H, Mitsushima T, Yamaji Y, Okamoto $M$, Wada R, Kokubo T, Doi $\mathrm{H}$, Yoshida H, Kawabe T, Omata M: Predicting the development of gastric cancer from combining Helicobacter pylori antibodies and serum pepsinogen status: a prospective endoscopic cohort study. Gut 2005;54:764768.

7 Fukase K, Kato M, Kikuchi S, Inoue K, Uemura N, Okamoto S, Terao S, Amagai K, Hayashi S, Asaka M: Effect of eradication of Helicobacter pylori on incidence of metachronous gastric carcinoma after endoscopic resection of early gastric cancer: an open-label, randomised controlled trial. Lancet 2008; 372:392-397.

8 Mizuno S, Miki I, Ishida T, Yoshida M, Onoyama M, Azuma T, Habu Y, Inokuchi $\mathrm{H}$, Ozasa K, Miki K, Watanabe Y: Prescreening of a high-risk group for gastric cancer by serologically determined Helicobacter pylori infection and atrophic gastritis. Dig Dis Sci 2010;55:3132-3137.

9 Ichinose M, Miki K, Furihata C, Kageyama T, Hayashi R, Niwa H, Oka H, Matsushima T, Takahashi K: Radioimmunoassay of serum group I and group II pepsinogens in normal controls and patients with various disorders. Clin Chim Acta 1982;126:183-191.

10 Samloff IM, Varis K, Ihamaki T, Siurala M, Rotter JI: Relationships among serum pepsinogen I, serum pepsinogen II, and gastric mucosal histology. A study in relatives of patients with pernicious anemia. Gastroenterology 1982;83:204-209.

11 Miki K, Ichinose M, Furihata C, Niwa H, Oka $\mathrm{H}$, Oda T, Matsushima T: Potential peptic activity of pepsinogen of human gastroduodenal mucosa determined by fluorescent microassay method using succinyl albumin. Clin Chim Acta 1982;121:337-344.

12 Miki K, Ichinose M, Ishikawa KB, Yahagi N, Matsushima M, Kakei N, Tsukada S, Kido M, Ishihama S, Shimizu Y, et al: Clinical application of serum pepsinogen I and II levels for mass screening to detect gastric cancer. Jpn J Cancer Res 1993;84:1086-1090.

13 Miki K, Ichinose M, Kakei N, Yahagi N, Matsushima M, Tsukada S, Ishihama S, Shimizu Y, Suzuki T, Kurokawa K, Takahashi K: The clinical application of the serum pepsinogen I and II levels as mass screening method for gastric cancer; in Takahashi K (ed): Aspartic Proteinases: Structure, Function, Biology and Biomedical Implications. New York, Plenum Press, 1995, pp 139-143.

14 Watanabe Y, Kurata JH, Mizuno S, Mukai M, Inokuchi $\mathrm{H}$, Miki K, Ozasa K, Kawai K: Helicobacter pylori infection and gastric cancer. A nested case-control study in a rural area of Japan. Dig Dis Sci 1997;42:13831387.

15 Ohata H, Kitauchi S, Yoshimura N, Mugitani K, Iwane M, Nakamura H, Yoshikawa A, Yanaoka K, Arii K, Tamai H, Shimizu Y, Takeshita T, Mohara O, Ichinose M: Progres- sion of chronic atrophic gastritis associated with Helicobacter pylori infection increases risk of gastric cancer. Int J Cancer 2004;109: 138-143.

16 Yoshida T, Kato J, Inoue I, Yoshimura N, Deguchi H, Mukoubayashi C, Oka M, Watanabe M, Enomoto S, Niwa T, Maekita T, Iguchi M, Tamai H, Utsunomiya H, Yamamichi N, Fujishiro M, Iwane M, Takeshita T, Ushijima T, Ichinose M: Cancer development based on chronic active gastritis and resulting gastric atrophy as assessed by serum levels of pepsinogen and Helicobacter pylori antibody titer. Int J Cancer 2014;134:14451457.

17 Sasazuki S, Inoue M, Iwasaki M, Otani T, Yamamoto S, Ikeda S, Hanaoka T, Tsugane S; Japan Public Health Center Study Group: Effect of Helicobacter pylori infection combined with CagA and pepsinogen status on gastric cancer development among Japanese men and women: a nested case-control study. Cancer Epidemiol Biomarkers Prev 2006;15: 1341-1347.

18 Yanaoka K, Oka M, Mukoubayashi C, Yoshimura N, Enomoto S, Iguchi M, Magari $\mathrm{H}$, Utsunomiya $\mathrm{H}$, Tamai $\mathrm{H}$, Arii $\mathrm{K}$, Ohata $\mathrm{H}$, Fujishiro M, Takeshita T, Mohara O, Ichinose M: Cancer high-risk subjects identified by serum pepsinogen tests: outcomes after 10-year follow-up in asymptomatic middle-aged males. Cancer Epidemiol Biomarkers Prev 2008;17:838-845.

19 Watanabe M, Kato J, Inoue I, Yoshimura N, Yoshida T, Mukoubayashi C, Deguchi H, Enomoto S, Ueda K, Maekita T, Iguchi M, Tamai $\mathrm{H}$, Utsunomiya $\mathrm{H}$, Yamamichi $\mathrm{N}, \mathrm{Fu}-$ jishiro M, Iwane M, Tekeshita T, Mohara $\mathrm{O}$, Ushijima T, Ichinose M: Development of gastric cancer in nonatrophic stomach with highly active inflammation identified by serum levels of pepsinogen and Helicobacter pylori antibody together with endoscopic rugal hyperplastic gastritis. Int J Cancer 2012;131: 2632-2642.
Facts About a New Gastric Cancer

Screening - The ABC Method
Digestion 2016;93:13-18 DOI: $10.1159 / 000441742$ 
20 Masuyama H, Yoshitake N, Sasai T, Nakamura T, Masuyama A, Zuiki T, Kurashina K, Mieda M, Sunada K, Yamamoto H, Togashi K, Terano A, Hiraishi H: Relationship between the degree of endoscopic atrophy of the gastric mucosa and carcinogenic risk. Digestion 2015;91:30-36.

21 Miki K: Gastric cancer screening by combined assay for serum anti-Helicobacter pylori IgG antibody and serum pepsinogen levels - 'ABC method'. Proc Jpn Acad Ser B Phys Biol Sci 2011;87:405-414.

22 Hisamichi S: Screening for gastric cancer. World J Surg 1989;13:31-37.
23 Bureau of Social Welfare and Public Health, Tokyo Metropolitan Government. http:// www.fukushihoken.metro.tokyo.jp/kensui/ gan/toukei/data/kako.html (in Japanese).

24 Nagata Y, Yamaguchi Y, Hiratsuka R, Tanaka U, Takahara T, Hirata K, Kawase Y, Tominaga T, Takeuchi S, Moritani M, Sakagami S, Ishida $S$ : The efficacy of new gastric cancer screening, the $\mathrm{ABC}$ method, in Nishitokyo city. J Tokyo Med Assoc 2014;67:60-68.
25 Terasawa T, Nishida H, Kato K, Miyashiro I, Yoshikawa T, Takaku R, Hamashima C: Prediction of gastric cancer development by serum pepsinogen test and Helicobacter pylori seropositivity in Eastern Asians: a systematic review and meta-analysis. PLoS One 2014; 9:e109783.

26 Gotoda T, Ishikawa $\mathrm{H}$, Ohnishi $\mathrm{H}$, Sugano K, Kusano C, Yokoi C, Matsuyama Y, Moriyasu F: Randomized controlled trial comparing gastric cancer screening by gastrointestinal X-ray with serology for Helicobacter pylori and pepsinogens followed by gastrointestinal endoscopy. Gastric Cancer 2015; 18:605-611. 